\title{
Designing surveys for radiography and radiation therapy
}

\author{
M O'Connor and G Halkett
}

WA Centre for Cancer and Palliative Care, Curtin University of Technology, GPO Box U1987, Perth, Western Australia 6845, Australia. Correspondence to m.oconnor@curtin.edu.au

\begin{abstract}
Surveys using standardised questionnaires are frequently used in the health area for research, to gather information to inform or improve clinical practice and to gage perceptions or attitudes towards particular issues. As we begin to conduct more studies in the areas of radiography and radiation therapy we need to ensure that it is essential that we use reliable and valid research methods so that findings are robust and, importantly, useable in clinical practice. This review focuses on how to design the questionnaire and highlights some key rules to help avoid the many pitfalls in survey design. The importance of the planning stage and time spent reviewing and revising questions is emphasised and the benefits and challenges of using existing instruments are discussed. Questionnaire layout is addressed and also the wording of individual questions as both of these will affect response rates and how people interpret questions. Wording can be difficult and may take a few drafts to get right. Several examples are given to demonstrate the concepts. The key messages in this review are: spend time planning and make the questionnaire brief, easy and safe. Issues such as response rates, types of questions, how to test for reliability and validity; and how to analyse the data will be covered in a further review for The Radiographer.
\end{abstract}

Keywords: questionnaire design, questionnaire layout, survey research, wording of questions.

\section{Introduction}

As we recognise the need for research and begin to conduct more studies in the areas of radiography and radiation therapy, ${ }^{1,2,3,4,5}$ it is essential that we use reliable and valid research methods so that findings are robust and, importantly, useable in clinical practice. Surveys using standardised questionnaires are frequently used in the health area for research, to gather information to inform or improve clinical practice and to gauge perceptions or attitudes towards particular issues. This review provides a set of guidelines a checklist - to use when designing and developing a questionnaire. It also includes references to literature that will provide further details and a more in-depth look at each of these issues.

Surveys are extremely useful in that they provide large amounts of data in a short period of time. This information can be easily analysed to provide a cross-sectional snapshot about an area of interest including people's needs, views, perceptions and behaviours. ${ }^{6}$ This information can be obtained quickly and inexpensively compared to face-to-face in-depth interviews, which are labour intensive and usually involve travel costs. ${ }^{7}$ However, having a large amount of data is not enough to make meaningful conclusions. We need to be very careful at the design stage because participants are completing the questionnaire on their own with no guidance or explanation. Researchers need to follow some key rules and spend time at the planning stage reviewing and revising questions in order to avoid the many pitfalls in survey design.

\section{Existing instruments}

An instrument, which can be used to answer the research questions may already have been developed. ${ }^{6}$ The benefits of using an existing instrument is that the instrument will have been tried and tested and will have established reliability and validity. There may also be an opportunity to compare findings with existing normative data e.g. data obtained using the same instru- ment from a previous decade or another geographical area will enable researchers to look at points of difference and similarity. ${ }^{8}$ Problems using an established instrument include not being able to change or modify the questions; having to compromise and look at something slightly different to what was planned; costs may be involved; and the instrument may be hard to get hold of. ${ }^{9}$ Notwithstanding these challenges it is always worth looking at what instruments are available in an area of interest. For example, if the research question relates to patient anxiety, there are a number of instruments that are available to measure this. It might be appropriate to use the Hospital Anxiety and Depression Scale $^{10}$ or the State Trait Anxiety Scale ${ }^{11}$. If we were interested in determining patients' needs we might use something like the supportive care needs survey. ${ }^{12}$ In radiography and radiation therapy there may not be a survey available for what we wish to measure. It may therefore be beneficial to develop a new survey and test it for validity and reliability. Other people in the profession can then use it as a reliable measure in their departments.

\section{First steps}

An essential first step is to be clear about what you want to know/discover. An unambiguous research question - e.g. what are people's attitudes towards receiving radiotherapy? - will inform the questionnaire questions and help avoid irrelevant questions. ${ }^{13}$ A common pitfall in designing a questionnaire is to include everything "just in case." If the research is looking at group differences - e.g. are younger patients less anxious than older patients when they are referred for a CT Scan? - questions about income or educational level are extraneous. The message is: questionnaire questions need to relate to the overall focus and the overarching research question. It is also important even at this early stage to think about how you are going to analyse the data and what comparisons you are going to make. 


\section{Layout}

Layout is important as people are put off a questionnaire that is crowded with messy pages. The key is to avoid mess, clutter and confusing layouts - a simple layout with headings to guide the reader makes completing a questionnaire easier, which in turn increases the response rate i.e. the proportion of people returning the questionnaire. ${ }^{14}$ Grouping similar questions together and where possible, grouping questions with similar ways of responding together also helps, e.g. all yes/no questions together, all questions using a scale and all open-ended questions. The rule is to be consistent. ${ }^{15}$ Grouping similar items enables people to move quickly through the questionnaire without having to stop and think about what is wanted. Consistency also reduces the likelihood of errors. ${ }^{16}$ Even when trying to save space crowding questions on the page is counter-productive as space is needed to break up the questionnaire. The following knowledge questions are numbered, need the same response and they are lined up carefully.

1 I need radiation therapy because it will reduce the chance of recurrence

$$
\square \text { True } \square \text { True } \square \text { True }
$$

2 I will receive radiation therapy every day I receive treatment $\square$ True $\square$ True $\square$ True

The more attractive a questionnaire is the easier it will be to complete. However, this needs to be balanced by simplicity - e.g. a questionnaire that includes pictures or patterns can be diverting. A 2002 meta-analysis $^{7}$ found that using coloured ink rather than blue or black ink increased response rates. However, this adds to the expense. Alternatively, it might be possible to use different coloured paper. In summary, pedantic attention to detail is essential to ensure people feel the questionnaire will be easy and quick to complete. The layout is important for both clinicians and patients who might be asked to complete the questionnaire. Everyone is time limited.

\section{Structure}

A preamble is needed to tell people what the questionnaire is for. A friendly, concise introduction to the questionnaire is vital to get people interested. Give a brief description of the aims of questionnaire and state the benefits to participants and people generally. This should be written in very basic language. Anything that adds to the legitimacy of the questionnaire should be included - a logo or reference to an organisation will help people feel safe i.e. that the information will be used appropriately. Response rates to surveys ${ }^{7}$ from universities, for example, are usually higher than those from commercial agencies. Clear instructions are also needed for each part of a questionnaire. These need to be worded concisely and carefully to avoid any ambiguity. Examples help because readers can see clearly what needs to be done. Use an example from a different area or domain to avoid priming the participant to respond in a particular way.

You need to include an information sheet, which also needs to be brief and clear. Outline the aims of the survey and any anticipated benefits to the person, profession and community. Ethical considerations need to be highlighted also - information needs to be treated confidentially and responses need to remain anonymous.

Demographic information is best placed at the beginning of the questionnaire or at the end. The logic for placing demographic questions at the end is that people will be used to answering questions and will not hesitate to give personal details. ${ }^{17}$ Roberson and Sundstrom ${ }^{18}$ found that response rates were higher when demographic items were placed at the end of the questionnaire even though there were only four background items. The logic for placing them at the beginning is to get background questions "out of the way" before asking for views and attitudes. The wording of these questions is also important because people might be sensitive about their age or other details.

Ask the most relevant questions first to capture interest and to maximise the likelihood of people responding. Topic order has a significant impact on return rates. In one study when items were arranged according to relevance to participants the response rate was $18 \%$ higher than when items were randomly ordered. ${ }^{18}$ Sensitive questions can be asked later in the sequence so that participants become familiar with the questionnaire format and used to responding. ${ }^{13}$ Following this argument, if we were to ask about patient anxiety we would put these questions later rather than sooner. However, we also need to be mindful that we want these items answered so it is better not to put them at the end of the questionnaire. Alternatively, a funnel sequence can be used so that general questions are asked first, then less general questions and finally specific questions. ${ }^{19}$

Try and avoid "leaps" of topics in each section. If a section includes a group of questions on feelings towards a particular issue do not include questions about behaviour or about feelings towards a different issue. The rule once again is consistency. People are cognitively "lazy" 20 and prefer not to have to shift their thinking suddenly from one area to another. A logical flow guides the participant through the questionnaire. For example, in designing a questionnaire about advanced practice it is necessary to think about specialities within advanced practice and link these together rather than jumping between sections.

\section{Designing questions}

The wording of individual questions will greatly affect response rates and also how people interpret the questions. ${ }^{21}$ This wording can be difficult and may take a few drafts to get right. How we ask questions in conversation or during an interview is very different to how we need to ask questions in a questionnaire. The following is a list of common pitfalls (please see $e^{13,17,22}$ for further discussion of these issues).

1 Avoid repetition - in everyday conversations we often ask the same question in several different ways. In designing a questionnaire we need to avoid this.

2 Ask - "Is this necessary?" Only include what you need and what you will use. E.g. If you are not going to use income then don't include it. Income can be a sensitive issue and best avoided if it is just for interest rather than to be used in the analysis. Each question needs to be reviewed to see if it is necessary and, importantly, analysable and useable. If not then it is taking up valuable space.

3 Clarity is important. Avoid ambiguity - questions should have a full range of mutually exclusive options. The question below is ambiguous as someone could live on a farm in the country.

"Where did you grow up?"

a country

$b$ farm

c city

For radiography:

What type of imaging did you undergo? $\square$ x-ray $\square$ CT $\square$ MRI 
Patients may have had all of these scans recently or may not know the differences between them.

Further pitfalls in terms of clarity are: vague questions and questions that are too general. Questions need to be specific and clear.

The question "Are you satisfied with work?" could be broken down further into aspects of work such as working environment, pay, colleagues, managerial support and stress levels.

4 Avoid leading questions - leading questions prime the participant to respond in a certain way. This prompting can greatly influence how people will respond.

"How necessary do you think a postgraduate specialisation in emergency radiography is?" This question implies that a postgraduate specialisation is good and that the participant just needs to say how good it is. This is also important in introductory statements. E.g. "Radiation therapists have recently received training to provide a better service for patients - this survey asks your views about this improved service" leads the participant to think about an improved service rather than asking the participant if training has had an impact on service delivery or has improved the service.

5 We need to consider how language is used. Keep questions short and simple - use words such as "help" rather than "facilitate," "buy" rather than "purchase," or "think" rather than "cogitate". Language should not be bombastic! Also, avoid complex questions.

"Do you think the staff members in this clinic, or centre, always conduct themselves with a modicum of decorum when carrying out their duties in delivering a quality service?"

Keep language non-threatening and avoid emotionally laden language. E.g. "How terrified are you about your CT scan?" However, this needs to be balanced with the need to use appropriate terms and to avoid euphemisms. E.g. if you are asking questions about the use of funeral services you would not use terms such as "passed away" or "passed on". You would need to use the direct terms such as "death" and "died". Also need to make sure terms are defined for patients - e.g. define the terms "planning" and "CT" for patients.

Try and use familiar words and expressions. If working with a particular sub-group, such as a professional group, be heedful of their use of language and also be aware that certain terms have different meanings for different groups. E.g. for a statistician "a significant result" has a specific statistical meaning. This is where pre-testing for meaning and understanding becomes very important.

Avoid abbreviations even when you know they will be familiar to participants. This will minimise the potential for misunderstanding. E.g. "Do you think Australian CPD requirements are adequate?"

This is better: Can you outline below the benefits of cone beam computed tomography $(C B C T)$ ?"

6 Avoid double-barrelled questions. The following questions all include two questions and should be split.

"Do you think transport to the hospital, if you used it, is adequate?"

"Do you like the layout and design of the department?"

"Were you satisfied with the quality of our communication and treatment?"
7 Use all available alternatives

"What qualifications do you have?

A Certificate

B Diploma

C Degree

These options are not exhaustive. Another viable option is: "Other, please state

8 Don't make assumptions - the following question assumes the participant has health insurance.

"Are you satisfied with your current health insurance?"

9 Where possible avoid "branching" or filters. However, if these are inevitable use very carefully and check that each one is clear. E.g.

Do you currently have a life insurance policy?

$\square$ Yes - if yes, please go to question 3

$\square$ No - if no please go to question 4

\section{Q.3 How much is your annual life insurance premium?}

Alternatively, these questions could be rewritten as one question that applies to everyone:

How much did you spend last year for life insurance?

(write 0 if nothing)

\section{When not to use a questionnaire}

Notwithstanding the many benefits of using a cross sectional survey design, there are times when another approach may be more appropriate. Questionnaires generate a lot of data and these data need to be managed very carefully. Focussing on what is needed not what may be useful helps but an analysis plan is essential i.e. what analyses will be used to answer what questions $?^{22}$

Questionnaires may not be the best method for eliciting answers to sensitive questions. Response rates to surveys asking for sensitive information are typically low, ${ }^{7}$ so if we are asking about sexuality or bereavement, we may be better to use face-to-face interviews so that trust and rapport can be established. Interviews may also be more useful when we need elaboration or supplementary information or an insight into how people feel about certain issues. ${ }^{23}$ Questions deliver a lot of information very quickly but this information will lack depth. Interviews are more likely to deliver rich data. We might survey patients about their overall experience and then interview a sample of them to find out more about what their treatment was like or how they were feeling. ${ }^{24}$

All the guidelines outlined above are designed to minimise misinterpretation but there is always the possibility of ambiguity and differences in interpretation. Questionnaires also often have missing data - questions or sections may not be completed due to oversight, lack of understanding, people not wanting to answer such questions or it could be something simple like turning over two pages at once.

\section{In conclusion}

The key messages in this review are: spend time planning and take a reductionist approach i.e. make it brief, make it easy and make it safe. This review focussed on how to design the questionnaire, which is only half the story. Issues such as response rates, types of questions, how to test for reliability and validity; and how to analyse the data will be covered in a further review, which will be published in the next issue of The Radiographer. 


\section{The authors}

Moira O'Connor BA (Hons), MSc, PhD

Senior Research Fellow

Georgia Halkett PhD, FIR, BMedRad(Hons)

Senior Research Fellow

\section{References}

1 Catton J, Catton P. Research and the medical radiation technologists. Can J Med Radiat Technol 1999; 30 (1): 35-44.

2 Nixon S. Professionalism in radiography. Radiography 2001; 7 (1): 31-5.

3 Scutter S, Halkett G. Research attitudes and experiences of radiation therapists. The Radiographer 2003; 50 (2): 69-71.

4 Harnett N, Palmer C, Bolderston A, Wenz J, Catton P. The scholarly radiation therapist. Part one: charting the territory. J Radiother Pract 2008; 7: 99-104.

5 Cox J, Halkett GKB, Anderson C. Research interests identified at the coalface: initial Delphi analysis of Australian radiation therapists' perspectives. The Radiographer 2009; 56 (2): 9-14.

6 Roberts K, Taylor B. Nursing research processes: An Australian perspective. 2nd ed. Victoria: Thomson Learning; 2002.

7 Edwards P, Roberts I, Clarke M, DiGuiseppi C, Pratap S, Wenyz R, Kwan I. Increasing response rates to postal questionnaires: systematic review. $B M J$ 2002; 324: 1.

8 Ciccetti DV. Guidelines, criteria and rules of thumb for evaluating normed and standardized assessment instruments in psychology. Psychol Assess 1994; 6 (4): 284-90.

9 Church AH, Waclawski J, Kraut AI. Understanding and using organizational surveys: a seven step process. New York (NY): Jossey-Bass; 2001.

10 Zigmund AS, Snaith RP. The hospital anxiety and depression scale. Acto Psychiatr Scand 1983; 67: 361-70.

11 Spielberger C, Gorsuch R, Lushene R. Manual for the State-Trait Anxiety Inventory. Palo Alto (CA): Consulting Psychologists Press; 1970.
12 Sanson-Fisher R, Girgis A, Boyes A, Bonevski B, Burton L, Cook P. The unmet supportive care needs of patients with cancer. Cancer 2000; 88 (1); 226-37.

13 Williams A. How to....write and analyse a questionnaire. J Orthod 2003; 30 245-52.

14 Mayer CS, Piper C. A note on the importance of layout in self-administered questionnaires. J Mark Res 1982; 19 (3): 390-91.

15 Breakwell GM, Hammond S, Fife-Schaw C, Editors. Research methods in psychology. 2nd ed. Thousand Oaks (CA): Sage Publications Ltd; 2000.

16 Schneider Z, Elliott D, LoBiondo-Wood G, Haber J. Nursing research: Methods, critical appraisal and utilization. 2nd ed. NSW: Elsevier; 2004.

17 Groves RM, Floyd J, Couper MP, Lepkowski JM, Singer E, Tourangeau R. Survey Methodology. 2nd ed. Hoboken (NJ): John Wiley \& Sons Inc; 2009.

18 Roberson MT, Sundstrom E. Questionnaire design, return rates, and response favourableness in an employee attitude questionnaire. J Appl Psychol 1990; 75 (3): 354-57.

19 Black N, Brazier J, Fitzpatrick R, Reeves B. Designing and using patient and staff questionnaires. In: Black N, Brazier J, Fitzpatrick R, Reeves B. Health services research methods-a Guide to best practice. London: BMJ Books; 1998.

20 Garcia-Retamero R, Takezawa M, Galesic M. Simple mechanisms for gathering social information. New Ideas Psychol 2010; 28 (1): 49-63.

21 Larsen JD, Mascharka C, Toronski C. Does the wording of the question change the number of headaches people report in a health questionnaire? Psychological Record 1983; 3: 423-27.

22 Central Public Health Network. Key guidelines for the development of questionnaires. Queensland: Queensland Health Department; 2006.

23 Babbie ER. The practice of social research. 12th ed. Belmont (CA): Wadsworth; 2010.

24 Fowler FJ. Survey Research Methods. 3rd ed. Thousand Oaks (CA): Sage Publications Inc; 2002. 